\title{
Fractional Quantum Hall States in Graphene
}

\author{
Ahmed Jellal $^{a, b} \cdot *$ and Bellati Malika ${ }^{b} \oplus$ \\ ${ }^{a}$ The Abdus Salam International Centre for Theoretical Physics, \\ Strada Costiera 11, 34014 Trieste, Italy \\ ${ }^{b}$ Theoretical Physics Group, Faculty of Sciences, Chouaïb Doukkali University, \\ Ibn Maâchou Road, PO Box 20, 24000 El Jadida, Morocco
}

\begin{abstract}
We quantum mechanically analyze the fractional quantum Hall effect in graphene. This will be done by building the corresponding states in terms of a potential governing the interactions and discussing other issues. More precisely, we consider a system of particles in the presence of an external magnetic field and take into account of a specific interaction that captures the basic features of the Laughlin series $\nu=\frac{1}{2 l+1}$. We show that how its Laughlin potential can be generalized to deal with the composite fermions in graphene. To give a concrete example, we consider the $S U(N)$ wavefunctions and give a realization of the composite fermion filling factor. All these results will be obtained by generalizing the mapping between the Pauli-Schrödinger and Dirac Hamiltonian's to the interacting particle case. Meantime by making use of a gauge transformation, we establish a relation between the free and interacting Dirac operators. This shows that the involved interaction can actually be generated from a singular gauge transformation.
\end{abstract}

\footnotetext{
*ajellal@ictp.it, jellal.a@ucd.ac.ma
}

${ }^{\dagger}$ mbelati@gmail.com 


\section{Introduction}

Nowadays the observation of the famous quantum Hall effect (QHE) [1] does not remain at the stage of the semiconductors, but it can be seen in other materials. This is for example the case of graphene, which is a projected graphite of the group symmetry $C_{3}$ into two-dimensional spaces. When such system is submitted to a perpendicular magnetic field, it appears an anomalous integer Hall conductivity [2, 3] that is a manifestation of the relativistic particle motions. It is originated from different sources, which are the four-fold spin/valley and the Berry phase due to the pseudospin (or valley) precision when a massless (chiral) Dirac particle exercises cyclotron motion. This result was theoretically suggested by two groups [4, 5] independently.

The emergence of QHE in graphene during 2005 offered a laboratory for different investigations and applications of some mathematical formalism. Many of them brought from the QHE studies in semiconductors and used the Dirac operator as the starting point instead of the Landau Hamiltonian. Indeed, different questions have been partially or completely solved but still some attentions to be paid to others. This concerns an eventual fractional quantum Hall effect (FQHE) [ 6] in graphene and related issues. Such interest can be linked to the fact that FQHE is a fascinating subject because starting from its appearance is still capturing a great attention. This is due to its relations to different areas of physics and mathematics.

There are many interesting theories have been appeared dealing with some problems related to the anomalous FQHE, for instance one may refer to the papers [7, 8, 9, 10]. In particular, based on different arguments some wavefunctions have been suggested as good candidates to describe the subject [11, 12, 13. In fact, some of them used the composite fermion approach [14] and other made a straightforward generalization of the Halperin theory [15] for non-polarized spin. However, some wavefunctions can be linked to each other and recovered from a general proposal as has been seen in [13].

Another theory was proposed by the first author [13] who studied the effect exhibited by the relativistic particles living on two-sphere $\mathbb{S}^{2}$ and submitted to a magnetic monopole. In fact, he started by establishing a direct connection between the Dirac and Landau operators through the Pauli-Schrödinger Hamiltonian $H_{\mathrm{s}}^{\mathrm{SP}}$. This was helpful in the sense that the Dirac eigenvalues and eigenfunctions were easily derived. In analyzing $H_{\mathrm{s}}^{\mathrm{SP}}$ spectrum, he showed that there is a composite fermion nature supported by the presence of two effective magnetic fields. For the lowest Landau level (LLL), he argued that the basic physics of graphene is similar to that of two-dimensional electron gas, which is in agreement with the planar limit. For the higher Landau levels, he proposed a $S U(N)$ wavefunction for different filling factors that captures all symmetries. Focusing on the graphene case, i.e. $N=4$, he gave different configurations those allowed to recover some known results.

Motivated by different analysis concerning the anomalous FQHE and in particular [13], we develop a real approach in order to describe the subject. Taking advantage of our knowledge about the phenomena in semiconductors, we introduce some effective interactions as relevant ingredient in forming the Hamiltonian system. For this, we consider $M$-particles in the presence of a perpendicular magnetic field $B$ where the interaction is taken to be two and three-body types. These have been successfully applied to the conventional FQHE, for instance one may see [16, 17]. Based on the connection between the Pauli-Schrödinger and Dirac Hamiltonian's, we construct an appropriate Dirac operator 
that captures the basic feature of the interacting system and where the Laughlin wavefunction [18] is its groundstate.

Because of the basic physics is the same in both systems: semiconductors and graphene for the Laughlin series, we start by establishing the corresponding effective potential. It will be generalized to describe the composite fermions in graphene [19] of filling factor $\nu_{\mathrm{cf}}=4\left(n+\frac{1}{2}\right)$, with $n=0, \pm 1, \pm 2, \cdots$. This of course captures the physics behind the fractional filling factors beyond Laughlin series $\frac{1}{2 l+1}$, with $l$ is integer, for FQHE in graphene. Moreover, we return to the $S U(N)$ wavefunctions [13] to firstly give a concrete example showing a particular type of potential. Secondly, we show how the corresponding filling factor can be linked to that generated from the composite fermions pictures [19]. Different discusions will be reported elsewhere about the matter.

The present paper is organized as follows. In section 2 , in order to show the difference between the interacting and free particles cases, we start by studying a system without interaction. We make use an approach based on the mapping between different Hamiltonian's to get the corresponding spectrum and eigenfunctions. In section 3, for the later convenience, we give some discussions about the Laughlin potential that captures the interaction effect. We introduce two and three-body interactions and analyze the behaviour the new system in section 4. In particular, we show that the Laughlin wavefunction is a groundstate of the present system and derive the spectrum's for the excited states. We note that the interactions can be generated from a singular transformation and allow us to establish a relation between both Dirac systems: free and interacting. In section 5, we consider the composite Dirac fermions in terms of an appropriate Jain potential, which can be obtained by making use of straightforward generalization of the Laughlin one. In this framework, we analyze the $S U(N)$ wavefunctions and show its basic features in section 6 . Finally, we conclude and give some perspectives of the present work.

\section{Dirac Hamiltonian}

We start by developing our approach that will be subsequently generalized to the case of the interacting system. This concerns a mapping between different spectrum's: Landau, Pauli-Schrödinger and Dirac. Indeed, let us consider one-relativistic particle living on the plane $(x, y)$ in presence of a perpendicular magnetic field $B$. This can be described by

$$
H_{\mathrm{PS}}=\frac{1}{2 m}\left[\vec{\sigma} \cdot\left(\vec{P}-\frac{e}{c} \vec{A}\right)\right]^{2}
$$

where $\vec{\sigma}$ are the Pauli matrices and verify the usual relations, namely

$$
\left\{\sigma_{i}, \sigma_{j}\right\}=2 \delta_{i j}, \quad\left[\sigma_{i}, \sigma_{i}\right]=2 \epsilon_{i j k} \sigma_{k} .
$$

We will see how the Pauli-Schrödinger Hamiltonian $H_{\mathrm{PS}}$ can be used to get the Dirac operator for the present system. Specifically, it will be derived from the square root of $H_{\mathrm{PS}}$. This mapping is useful in sense that the Dirac eigenvalues and eigenfunctions will be easily obtained.

One way to establish the mentioned mapping is to express $H_{\mathrm{PS}}$ in terms of the Landau Hamiltonian, which describe free particle. This connection has an important advantage because it leads to exactly derive different quantities related to the subject. Thus, by choosing the symmetric gauge

$$
\vec{A}=\frac{B}{2}(-y, x)
$$


we show that $H_{\mathrm{PS}}$ can be mapped in the form

$$
H_{\mathrm{PS}}=\left(\begin{array}{cc}
H_{\mathrm{L}} & 0 \\
0 & H_{\mathrm{L}}
\end{array}\right)-\frac{B}{2}\left(\begin{array}{cc}
1 & 0 \\
0 & -1
\end{array}\right)
$$

where $H_{\mathrm{L}}$ is simply the Landau Hamiltonian and reads as

$$
H_{\mathrm{L}}=\frac{1}{2 m}\left(\vec{P}-\frac{e}{c} \vec{A}\right)^{2}
$$

which is extremely used in different contexts and in particular in analyzing QHE in the semiconductor systems. To easiest way to resort different spectrum is to write $H_{\mathrm{L}}$ in terms of the annihilation and creation operators as

$$
H_{\mathrm{L}}=\frac{1}{4}\left(a^{\dagger} a+a a^{\dagger}\right)
$$

where, in the complex notation $z=x+i y, a$ and $a^{\dagger}$ are given by

$$
\hat{a}=2 \frac{\partial}{\partial z}+\frac{B}{2} \bar{z}, \quad \hat{a}^{\dagger}=-2 \frac{\partial}{\partial \bar{z}}+\frac{B}{2}|z|^{2} .
$$

They verify the commutation relation

$$
\left[\hat{a}, \hat{a}^{\dagger}\right]=2 B .
$$

Since we are wondering to generalize different Hamiltonian's entering in the game, let us convert $H_{\mathrm{L}}$ into its analytical form. It is not hard to obtain

$$
H_{\mathrm{L}}=-\frac{1}{2}\left\{4 \frac{\partial^{2}}{\partial z_{i} \partial \bar{z}_{i}}-B\left(z \frac{\partial}{\partial z}-\bar{z} \frac{\partial}{\partial \bar{z}}\right)-\frac{B^{2}}{4} \bar{z}\right\} .
$$

Hereafter, we set the fundamental constants $(e, c, \hbar, m)$ to one. Clearly, the spectrum and eigenfunctions of $H_{\mathrm{PS}}$ can be derived from that of $H_{\mathrm{L}}$.

The above tools can be applied to analysis the anomalous QHE in graphene. Indeed in such systems, the two-Fermi points, each with a two-fold band degeneracy, can be described by a lowenergy continuum approximation with a four-component envelope wavefunction whose components are labelled by a Fermi-point pseudospin $= \pm 1$ and a sublattice forming an honeycomb. Specifically, the Hamiltonian for one-pseudospin component can be obtained from (4) under some consideration. This is

$$
H_{\mathrm{D}}=\frac{i}{\sqrt{2}} v_{\mathrm{F}}\left(\begin{array}{cc}
0 & \hat{a}^{\dagger} \\
-\hat{a} & 0
\end{array}\right) .
$$

where $v_{\mathrm{F}} \approx \frac{c}{100}$ is the Fermi velocity, which will be set to one, and the many-body effects are neglected. Its spectrum can be determined in a simple way if we introduce its square. This is

$$
H_{\mathrm{D}}^{2}=\frac{1}{2}\left(\begin{array}{cc}
\hat{a}^{\dagger} \hat{a} & 0 \\
0 & \hat{a} \hat{a}^{\dagger}
\end{array}\right) .
$$

It is related to the Pauli-Schrödinger Hamiltonian (44) up to some multiplicative constants. It is clear that, $H_{\mathrm{D}}^{2}$ is written in terms of the diagonal form of the Landau Hamiltonian (9). Therefore, it spectrum can easily be obtained.

We start by solving the eigenvalue equation

$$
H_{\mathrm{D}}^{2} \Psi=E_{\mathrm{D}}^{2} \Psi
$$


Since $H_{\mathrm{D}}^{2}$ has to do with the Landau Hamiltonian (9), the wavefunctions $\Psi$ should be written in an appropriate form. This is

$$
\Psi_{m, n}=\left(\begin{array}{c}
\psi_{m, n} \\
\psi_{m-1, n}
\end{array}\right)
$$

where the eigenfunctions $\psi_{m, n}$ are given by

$$
\psi_{m, n}(z, \bar{z})=\frac{(-1)^{m} \sqrt{B^{m} m !}}{\sqrt{2^{n+1} \pi(m+n) !}} z^{n} L_{m}^{n}\left(\frac{z \bar{z}}{2}\right) e^{-\frac{B}{4} z \bar{z}}, \quad m, n=0,1,2 \cdots .
$$

The corresponding Landau levels are given by

$$
E_{\mathrm{D}}^{2}(m)=B m
$$

We can show that the normalized eigenfunctions of $H_{\mathrm{D}}$ take the form

$$
\Psi_{m \neq 0, n}=\frac{1}{\sqrt{2}}\left(\begin{array}{c}
\psi_{|m|, n} \\
-i \psi_{|m|-1, n}
\end{array}\right)
$$

with the convention $\psi_{-1, n}=0$. Note that, the zero mode wavefunction is

$$
\Psi^{(0, n)}=\left(\begin{array}{c}
\psi_{0, n} \\
0
\end{array}\right)
$$

Their energy levels read as

$$
E_{\mathrm{D}}(m)= \pm \sqrt{B|m|}
$$

This makes a difference with respect to the Landau spectrum for the present system. In fact, it has no zero energy and is discrete as well, unlike (18). These what make the integer QHE is an unconventional effect in graphene.

The above analysis can easily be generalized to many-body system without interaction. This leads to a spectrum as sum over all single particle ones and eigenfunctions as tensor products. However, we will give a generalization of the system by introducing a kind of interaction that is behind FQHE in semiconductors and see what is its influence on graphene.

\section{Laughlin wavefunction}

To develop our main idea we need first to start from what we know so far about the Laughlin wavefunction. This latter is involving a Jastrow factor that is originated from a specific interaction between particles. In fact, it corresponds to an artificial model that captures the basic physics of the filling factor $\nu=\frac{1}{2 l+1}$. This is an typical example to get more general results, which concern the composite fermions as well as the $S U(4)$ wavefunctions for graphene. For our task it is necessary to start from the Laughlin theory for FQHE in graphene.

To talk about the Laughlin series for FQHE, it is convenient to introduce some discussions about LLL because it is a rich level and has many interesting properties. Indeed, in such level the Landau system behaves like a non-commutative one, which is governed by the commutation relation

$$
[z, \bar{z}]_{\mathrm{LLL}}=\frac{2}{B}
$$


This effect can be interpreted as follows. In the present level, the potential energy is strong enough than kinetic energy and therefore the particles are glue in the fundamental level. Using the same analysis as for the case of $H_{\mathrm{L}}$, is not hard to get a basis as set of the eigenstates. They are [20]

$$
|m\rangle_{\mathrm{LLL}}=\frac{1}{\sqrt{m !}}\left(\hat{a}^{\dagger} \mid \mathrm{LLL}\right)^{m}|0\rangle_{\mathrm{LLL}}
$$

where the annihilation and creation operators reduce now to

$$
\left.\hat{a}\right|_{\mathrm{LLL}}=\frac{B}{2} z,\left.\quad \hat{a}^{\dagger}\right|_{\mathrm{LLL}}=\frac{B}{2} \bar{z} .
$$

The projection into the complex plane leads to the eigenfunctions

$$
\Psi_{m}(z, \bar{z})=\sqrt{\frac{B^{m+1}}{2^{m+1} \pi m !}} z^{m} e^{-\frac{B}{4}|z|^{2}} .
$$

As we will see very soon, this can be generalized to get more interesting results. In particular, these will offer a way to get in contact with the Laughlin theory [18] for FQHE.

Returning now to discuss FQHE in graphene. In doing so, Let us consider $M$-particles in LLL, which of course means that all $m_{i}=0$ with $i=1, \cdots, M$ and each $m_{i}$ corresponds to the spectrum (16[18). The total wavefunction of zero-energy Landau level (17) can be written in terms of the Slater determinant. This is

$$
\psi(z, \bar{z})=\epsilon^{i_{1} \cdots i_{M}} z_{i_{1}}^{m_{1}} \cdots z_{i_{M}}^{m_{M}} \exp \left(-\frac{B}{4} \sum_{i}^{M}\left|z_{i}\right|^{2}\right)
$$

where $\epsilon^{i_{1} \cdots i_{M}}$ is the fully antisymmetric tensor and $m_{i}$ are integers. It is relevant to write this wavefunction as Vandermonde determinant. We have

$$
\psi(z, \bar{z})=\mathrm{const} \prod_{i<j}^{M}\left(z_{i}-z_{j}\right) \exp \left(-\frac{B}{4} \sum_{i}^{M}\left|z_{i}\right|^{2}\right) .
$$

The corresponding energy is

$$
E\left(m_{i}\right)=B\left(\sum_{i} m_{i}+M\right) .
$$

This can be interpreted by remembering the Laughlin wavefunction

$$
\psi_{\text {Laugh }}^{l}(z, \bar{z})=\prod_{i<j}^{M}\left(z_{i}-z_{j}\right)^{2 l+1} \exp \left(-\frac{B}{4} \sum_{i}^{M}\left|z_{i}\right|^{2}\right) .
$$

It is well-known that it has many interesting features and good ansatz to describe the fractional QHE at the filling factor $\nu=\frac{1}{2 l+1}$, with $l$ is integer value. It is clear that (24) is nothing but the first Laughlin state that corresponds to $\nu=1$. Actually, (24) is describing the first quantized Hall plateau of the integer QHE. It is also convenient for us to write (26) under another form. This is Note that (26) can also be written as

$$
|l\rangle_{\text {Laugh }}=\left\{\epsilon^{i_{1} \cdots i_{M}} z_{i_{1}}^{m_{1}} \cdots z_{i_{M}}^{m_{M}}\right\}^{2 l+1}|0\rangle .
$$

Consequently, the wavefunction for the particles in the graphene LLL are identical to those of the 2DEG LLL. We conclude that the basic physics in both systems is the same. More discussion about this issue can be found in 9 . 
As far as we know FQHE is a consequence of the interacting particles in the presence of an external magnetic field. Then, obviously the Laughlin wavefunction is a manifestation of a specific interaction type, which coded in the Jastrow factor. At this point, one may ask how does look like such interaction and to reply this question, let us write (26) as

$$
\psi_{\text {Laugh }}^{l}(z, \bar{z})=F^{\text {Laugh }}(z, \bar{z}) \exp \left(-\frac{B}{4} \sum_{i}^{M}\left|z_{i}\right|^{2}\right)
$$

where the function $F^{\text {Laugh }}(z, \bar{z})$ ant its anti-holomorphic partner are given by

$$
F^{\text {Laugh }}(z, \bar{z})=\prod_{i<j}^{M}\left(z_{i}-z_{j}\right)^{2 l+1}, \quad \bar{F}^{\text {Laugh }}(z, \bar{z})=\prod_{i<j}^{M}\left(\bar{z}_{i}-\bar{z}_{j}\right)^{2 l+1} .
$$

These are nothing but the results of a specific interaction, which generated from particles. To clarify this point, let us define two functions as the derivatives with respect to the variables $z$ and $\bar{z}$ of $F^{\text {Laugh }}$ and $\bar{F}^{\text {Laugh }}$, respectively. They are

$$
\left(V_{z}^{\text {Laugh }}\right)_{i}=-2 i \frac{\partial}{\partial z_{i}}\left(\ln F^{\text {Laugh }}-\ln \bar{F}^{\text {Laugh }}\right), \quad\left(V_{\bar{z}}^{\text {Laugh }}\right)_{i}=2 i \frac{\partial}{\partial \bar{z}_{i}}\left(\ln F^{\text {Laugh }}-\ln \bar{F}^{\text {Laugh }}\right) .
$$

Explicitely, the corresponding potential can be written as

$$
\left(V_{z}^{\text {Laugh }}\right)_{i}=-2 i(2 l+1) \sum_{j \neq i} \frac{1}{z_{i}-z_{j}}, \quad\left(V_{\bar{z}}^{\text {Laugh }}\right)_{i}=-2 i(2 l+1) \sum_{j \neq i} \frac{1}{\bar{z}_{i}-\bar{z}_{j}}
$$

which is resulting from of interaction of the $i^{\text {th }}$ particle with the remaining $j$ ones. These can also be identified to the components of the Chern-Simon potential, which has been employed at many occasions to study the conventional QHE. For instance, more discussion about such identification can be found in [17]. This of course open another way to describe the anomalous QHE by making use the field theory approach. Strictly speaking the present potential is, in reality, a part of an Hamiltonian whose groundstate is exactly the Laughlin wavefunction. Moreover, we show that how (30) can be generalized and used to discuss other filling factors beyond Laughlin states for FQHE in graphene. This mainly we will be done by adopting the composite fermion picture [14] and using the $S U(4)$ wavefunctions [13] as an illustration.

\section{Many-body interactions}

As claimed before we are wondering to investigate the basic feature of FQHE in graphene. In doing so, we have to take account of a kind of interaction. For this, we start with a Hamiltonian that has been considered at different occasions and is relevant to describe FQHE at the filling factor $\nu=\frac{1}{2 l+1}$. Further, we show that this will be applied to graphene and allow us to derive different results. Mainly, we apply the approach seen before for free relativistic particles to achieve our goal. In fact, we start from a Hamiltonian generalizing the Landau one to map a bridge between the Pauli-Schrödinger and Dirac Hamiltonian's for interacting particles. Of course, this will be done by constructing the appropriate annihilation and creation operators those will be used to build the excited states and derive the corresponding energies. 


\subsection{Excited states}

In the beginning, we start by resorting the spectrum for a system that include a specific interaction. This can be done by constructing an explicit model that has the Laughlin wavefunction as an exact groundstate. Let us particles living on the plane in the presence of an external magnetic field and involve some kind of interactions. When these are of two-body and three-body types, the present system can be described by the Hamiltonian

$$
\begin{aligned}
H_{\mathrm{L}}^{\mathrm{int}}= & \frac{1}{2} \sum_{i}^{M}\left(p_{i}-A_{i}\right)^{2}+\eta \sum_{i, j \neq i}^{M} \frac{1}{\left(z_{i}-z_{j}\right)}\left(2 \frac{\partial}{\partial \bar{z}_{i}}-i A_{z}\right)-\eta \sum_{i, j \neq i}^{M} \frac{1}{\left(\bar{z}_{i}-\bar{z}_{j}\right)}\left(2 \frac{\partial}{\partial z_{i}}-i A_{\bar{z}}\right) \\
& +2 \eta^{2} \sum_{i, j \neq i, k \neq i}^{M} \frac{1}{\left(z_{i}-z_{j}\right)\left(\bar{z}_{i}-\bar{z}_{k}\right)}
\end{aligned}
$$

where the coordinate $z_{i}=x_{i}+i y_{i}$ denote the position of the $i^{t h}$ particle and $\eta$ is the inverse of the Laughlin series, i.e. $\eta=2 l+1$ is an odd integer. The second and third terms in (32) are two-body interactions whereas the fourth one is a three-body type. In the symmetric gauge where the complex components of the vector potential $\vec{A}$ are

$$
A_{z}=-\frac{i}{2} B z, \quad A_{\bar{z}}=\frac{i}{2} B \bar{z}
$$

the Hamiltonian (32) takes the form

$$
\begin{aligned}
H_{\mathrm{L}}^{\mathrm{int}}= & -\frac{1}{2} \sum_{i}^{M}\left\{4 \frac{\partial^{2}}{\partial z_{i} \partial \bar{z}_{i}}-B\left(z_{i} \frac{\partial}{\partial z_{i}}-\bar{z}_{i} \frac{\partial}{\partial \bar{z}_{i}}\right)-\frac{B^{2}}{4} z_{i} \bar{z}_{i}\right\}+\eta \sum_{i, j \neq i}^{M} \frac{1}{\left(z_{i}-z_{j}\right)}\left(2 \frac{\partial}{\partial \bar{z}_{i}}-\frac{B}{2} z_{i}\right) \\
& -\eta \sum_{i, j \neq i}^{M} \frac{1}{\left(\bar{z}_{i}-\bar{z}_{j}\right)}\left(2 \frac{\partial}{\partial z_{i}}+\frac{B}{2} \bar{z}_{i}\right)+2 \eta^{2} \sum_{i, j \neq i, k \neq i}^{M} \frac{1}{\left(z_{i}-z_{j}\right)\left(\bar{z}_{i}-\bar{z}_{k}\right)} .
\end{aligned}
$$

It is obvious that when $\eta$ is switched off we get the Landau Hamiltonian for a free system of $M$ particles. Therefore, the presence of this parameter will play an important role in discussing different issues related to the anomalous FQHE.

To analyze the behaviour of interacting relativistic particles, it is relevant to derive the corresponding Dirac Hamiltonian. As far as we know, the easiest way to do is to adopt the technical used in section 2, which based on the mappings between three Hamiltonian's. More specifically, this can be done by making use of an algebraic method that requires to introduce the appropriate annihilation and creation operators. Indeed, in terms of the interaction they can be realized as

$$
\hat{A}_{i}=2 \frac{\partial}{\partial z_{i}}+\frac{B}{2} \bar{z}_{i}-2 \eta \sum_{j \neq i}^{M} \frac{1}{z_{i}-z_{j}}, \quad \hat{A}_{i}^{\dagger}=-2 \frac{\partial}{\partial_{\bar{z}_{i}}}+\frac{B}{2} z_{i}-2 \eta \sum_{j \neq i}^{M} \frac{1}{\bar{z}_{i}-\bar{z}_{j}} .
$$

It is not hard to show the commutation relation

$$
\left[\hat{A}_{i}, \hat{A}_{j}^{\dagger}\right]=2 B \delta_{i j}-4 \eta \sum_{i \neq j}^{M} \delta\left(z_{i}-z_{j}\right)-4 \eta \sum_{i \neq j}^{M} \delta\left(\bar{z}_{i}-\bar{z}_{j}\right)
$$


where for instance the second term is resulted by acting $\partial_{z_{i}}$ on the third term of $\hat{A}_{j}^{\dagger}$ and so on. These allow us to get a diagonal form of $H_{\mathrm{L}}^{\text {int }}$, such as

$$
H_{\mathrm{L}}^{\mathrm{int}}=\frac{1}{2}\left(\sum_{i}^{M} \hat{A}_{i}^{\dagger} \hat{A}_{i}+M B\right)-2 \eta \sum_{i \neq j}^{M} \delta\left(z_{i}-z_{j}\right) .
$$

Since $\delta\left(z_{i}-z_{j}\right)$ is irrelevant when acting on an antisymmetric wavefunction, it can be ignored. Moreover, $\hat{A}_{i}$ and $\hat{A}_{i}^{\dagger}$ satisfy the canonical harmonic oscillator commutation rules even for non-zero $\eta$. Hence, the Hamiltonian is exactly soluble for all the excited states. A basis of wavefunctions describing excitations of the $\nu=\frac{1}{\eta}$ states in the Laughlin theory is therefore given by

$$
\left|\left\{m_{i}\right\}, \eta\right\rangle_{\mathbf{L}}=\epsilon^{i_{1} \cdots i_{M}} \hat{A}_{i_{1}}^{m_{1}} \cdots \hat{A}_{i_{M}}^{\dagger_{M}}\left(\epsilon^{i_{1} \cdots i_{M}} \hat{A}_{i_{1}}^{\dagger^{0}} \cdot \hat{A}_{i_{M}}^{\dagger^{M-1}}\right)^{\eta-1}|0\rangle
$$

where $m_{i}$ are integers such that $0 \leq m_{1}<\cdots<m_{M}$. The corresponding eigenvalues are

$$
E_{\mathrm{L}}^{\mathrm{int}}\left(\left\{m_{i}\right\}, \eta\right)=B\left(\sum_{i}^{M} m_{i}+\frac{1}{2} M[(\eta-1)(M-1)+1]\right) .
$$

For later convenience, let us project these states on the complex plane. Indeed, by requiring that $m=i-1$, we end up with the wavefunction

$$
\psi_{\mathrm{L}}^{l}(z, \bar{z})=\prod_{k<l}^{M}\left(z_{k}-z_{l}\right) \prod_{i<j}^{M}\left(z_{i}-z_{j}\right)^{2 l+1} \exp \left(-\frac{B}{4} \sum_{i}^{M}\left|z_{i}\right|^{2}\right) .
$$

An interesting result can immediately be noticed. Indeed, now it is obvious to see that the Laughlin wavefunction is an exact groundstate of (34) and corresponds to the energy $\frac{M B}{2}$. More precisely, it is easy to check that $\hat{A}_{i}$ annihilates $|\eta\rangle_{\text {Laugh }} \equiv|l\rangle_{\text {Laugh }}$, namely

$$
\hat{A}_{i}|\eta\rangle_{\text {Laugh }}=0, \quad \forall i .
$$

This is nothing but the lowest Landau level condition. It is clear that for $\eta=1$ we recover the fully occupied state seen before. This show that the present model captures some basic features of the phenomenon.

In the next, we will show how the above tools can be used to describe FQHE in graphene. In the beginning we start by establishing the appropriate background to do our task. In particular, we determine the Dirac Hamiltonian and related matters for the present system.

\subsection{Spinors}

To apply the technical used before, it is convenient to write down the Pauli-Schrödinger Hamiltonian $H_{\mathrm{PS}}^{\mathrm{int}}$ for interacting particles in the presence of $B$. The easiest way to do is to give its expression in terms of the Hamiltonian $H_{\mathrm{L}}^{\mathrm{int}}$. The advantage of this mapping is make use an algebraic method to diagonalize and therefore derive the associate spectrum. This will be helpful in establishing the corresponding Dirac formalisms.

One way to write down $H_{\mathrm{PS}}^{\mathrm{int}}$ is to generalize the Hamiltonian (41) for free system to the interaction case. In doing so, we get the form

$$
H_{\mathrm{PS}}^{\mathrm{int}}=\left(\begin{array}{cc}
H_{\mathrm{L}}^{\mathrm{int}} & 0 \\
0 & H_{\mathrm{L}}^{\mathrm{int}}
\end{array}\right)+\left(\begin{array}{cc}
2 \eta \sum_{i \neq j}^{M} \delta\left(z_{i}-z_{j}\right) & 0 \\
0 & -2 \eta \sum_{i \neq j}^{M} \delta\left(\bar{z}_{i}-\bar{z}_{j}\right)
\end{array}\right)-\frac{1}{2} M B\left(\begin{array}{cc}
1 & 0 \\
0 & -1
\end{array}\right) .
$$


This is actually involving three matrices and the second one is particular. On the other hand, it can be identified to that used by Koshino and Ando [21] in discussing the diamagnetism in disordered graphene in arbitrary magnetic fields. However, in our case it be ignored since does not affect the antisymmetric wavefunction.

To start talking about different issues, we need first to resort the spectrum as well as the eigenstates of $H_{\mathrm{PS}}^{\mathrm{int}}$. Clearly, these matters can be easily derived to get the excited states as spinors

$$
|\{m\}, \eta\rangle_{\mathrm{PS}}=\left(\begin{array}{c}
\left|\left\{m_{i}\right\}, \eta\right\rangle_{\mathrm{L}} \\
\left|\left\{m_{i}-1\right\}, \eta\right\rangle_{\mathrm{L}}
\end{array}\right)
$$

where the eigenstates $\left|\left\{m_{i}\right\}, \eta\right\rangle_{\mathrm{L}}$ are those for the Hamiltonian (37), with the convention $|\{-1\}, \eta\rangle$ is a null state. The corresponding eigenvalues are

$$
E_{\mathrm{PS}}^{\mathrm{int}}(m, \eta)=B\left(\sum_{i=1}^{M} m_{i}+\frac{1}{2}(\eta-1) M(M-1)\right) .
$$

We have some remarks in order. For $m_{i}=0$ and $\eta=1$, there is a zero mode energy that corresponds to a fundamental state. However, for the case where $m_{i}=0$ and $\eta \neq 1$, we end up with

$$
E_{\mathrm{PS}}^{\mathrm{int}}(m=0, \eta)=\frac{1}{2}(\eta-1) B M(M-1) .
$$

This captures the basic features of the excited states for the present system, which is exactly the LLL condition. It offer another discussion about spin as a degree of freedom and its influence on the discussion reported before. Specifically, its relation with the conventional QHE, but this is out of scope of the present work.

At this level more discussion can be reported on the spectrum and in particular the corresponding Hilbert space, which is a set of all states $\left|\left\{m_{i}\right\}, \eta\right\rangle_{\mathrm{PS}}$. It is invariant under the semi-direct group $G=U(1) \times \mathbb{C}$ and therefore for an unitary projective representation $T(g)$ of $G$ we have the invariance property

$$
T(g) H_{\mathrm{PS}}^{\mathrm{int}}=H_{\mathrm{PS}}^{\mathrm{int}} T(g), \quad \forall g \in G
$$

where an element of $G$ is a $2 \times 2$ matrix of the form

$$
g=\left(\begin{array}{ll}
a & b \\
0 & 1
\end{array}\right) .
$$

This representation can be further explored and then make a group theory approach to investigate the basic features of the present system. It is acting on the spinors trough the relation

$$
T(g)|\{m\}, \eta\rangle_{\mathrm{PS}}=j(g, z) g^{-1}|\{m\}, \eta\rangle_{\mathrm{PS}}
$$

where the quantity $g^{-1}|\{m\}, \eta\rangle_{\mathrm{PS}}$ is the pull-back of $|\{m\}, \eta\rangle_{\mathrm{PS}}$ through the mapping $z \mapsto g \cdot z$ such as

$$
g \cdot z=a z+b .
$$

The function $j(g, z)$ is and automorphic factor. One also can add more analysis about the incompressibility in terms of the area preserving diffeomorphism algebra that can be realized in terms of the ingredients of the present system. 


\subsection{Interacting Dirac particles}

The above mathematical background can be applied to analyze FQHE in graphene. For this, it is word while to establish a Dirac formalism for the interacting particles. One way to do this is to make an analysis based on the former approach for non-interacting particles. This requires an analogue Dirac operator in terms of the annihilation and creation operators.

Right now we have all materials needed to do our job. Indeed, the appropriate interacting operator can be written in similar way to (50). Thus for the $i^{\text {th }}$ particle, we have

$$
\left(H_{\mathrm{D}}^{\mathrm{int}}\right)_{i}=\frac{i}{\sqrt{2}}\left(\begin{array}{cc}
0 & \hat{A}_{i}^{\dagger} \\
-\hat{A}_{i} & 0
\end{array}\right)
$$

where $\hat{A}_{i}$ and $\hat{A}_{i}^{\dagger}$ are given in (35). To make a comparison with the Pauli-Schrödinger Hamiltonian, let us define the square of $H_{\mathrm{D}}^{\mathrm{int}}$. This is given by

$$
\left[\left(H_{D}^{\mathrm{int}}\right)_{i}\right]^{2}=\frac{1}{2}\left(\begin{array}{cc}
\hat{A}_{i}^{\dagger} \hat{A}_{i} & 0 \\
0 & \hat{A}_{i} \hat{A}_{i}^{\dagger}
\end{array}\right)
$$

Summing over all $M$-particles and if we forget about all constants entering in different Hamiltonian's, it is not hard to end up with the relation

$$
\left(H_{\mathrm{D}}^{\mathrm{int}}\right)^{2}=H_{\mathrm{PS}}^{\mathrm{int}}
$$

This equivalence shows that how the applied approach is relevant as well to analyze the interacting particles. Remember that $\delta\left(z_{i}-z_{j}\right)$ and $\delta\left(\bar{z}_{i}-\bar{z}_{j}\right)$ can be dropped because they do not affect the antisymmetric wavefunction. Therefore, we can carry out our previous study concerning free Dirac particles and its connection to FQHE on graphene to the present case and underline the difference.

It is clear that, $\left(H_{\mathrm{D}}^{\mathrm{int}}\right)^{2}$ is written in terms of the diagonal form of the Hamiltonian (32) and therefore its spectrum can easily be obtained. Then, the eigenstates of $H_{\mathrm{D}}^{\text {int }}$ take the form

$$
|\{m\} \neq 0, \eta\rangle_{\mathrm{D}}=\left(\begin{array}{c}
\left|\left\{\left|m_{i}\right|\right\}, \eta\right\rangle_{\mathrm{L}} \\
-i\left|\left\{\left|m_{i}\right|-1\right\}, \eta\right\rangle_{\mathrm{L}}
\end{array}\right)
$$

and the zero mode is given by

$$
|\{0\}, \eta\rangle_{\mathrm{D}}=\left(\begin{array}{c}
|\{0\}, \eta\rangle_{\mathrm{L}} \\
0
\end{array}\right)
$$

The corresponding eigenvalues are

$$
E_{\mathrm{D}}^{\mathrm{int}}\left(m_{i}, \eta\right)= \pm \sqrt{B\left|\sum_{i=1}^{M} m_{i}+\frac{1}{2}(\eta-1) M(M-1)\right|} .
$$

It can be projected on LLL that is realized by requiring that $m_{i}=0$. This operation leads to the lowest energy for the excited Dirac states, such as

$$
E_{\mathrm{D}}^{\mathrm{int}}\left(m_{i}=0, \eta\right)= \pm \sqrt{\frac{1}{2}(\eta-1) M(M-1)} .
$$

Clearly, for $\eta=1$ we get a zero energy mode. This is an agreement with the fully occupied state $\nu=1$ see before fore the relativistic particles.

To close this part, one can also report some discussions about the symmetry group that leaves invariance the Dirac Hamiltonian for the present case. These of course will offer a theory group approach to analyze the interacting relativistic particles. 


\subsection{Gauge transformation}

Sometimes it is relevant to make the appropriate transformations in order to get some information about a system under consideration. This has an interst in sense that we can derive the integrability of the system from its old partner. For this, we are going to establish a concrete relation between the free and interacting Dirac operators. This can be done by considering an appropriate singular gauge transformation that captures the basic features the interacting term involved in different Hamiltonian's see before. It can also be seen as a kind of bosonization of the present system, for this we may refer to the reference [16]. The relation can be served as a good candidate to test the previous analysis concerning the interacting particle case.

We start with a system of $M$-relativistic fermions of mass $m$ in the presence of a external magnetic field. Without interaction, this system is described by the total Dirac Hamiltonian in the complex coordinates, such as

$$
H_{\mathrm{D}}=\frac{i}{\sqrt{2}}\left(\begin{array}{cc}
0 & \sum_{i}^{M}\left(-2 \frac{\partial}{\partial \bar{z}_{i}}+\frac{B}{2} z_{i}\right) \\
-\sum_{i}^{M}\left(2 \frac{\partial}{\partial z_{i}}+\frac{B}{2} \bar{z}_{i}\right) & 0
\end{array}\right) .
$$

This of course an immediate generalization of the Hamiltonian for one free particle. Therefore, its spectrum is nothing but a summation over that of one particle and the wavefunctions are tensor product of one single eigenfunctions.

To explicitly determine the element that governs such transformation, we adopt the method used by Karabali and Sakita [16]. In doing so, let us consider two wavefunctions and establish a link between them. This can be done by considering a singular gauge transformation, such as

$$
\Psi\left(z_{1}, \cdots, z_{N}\right)=U \Phi\left(z_{1}, \cdots, z_{N}\right)
$$

where $\Psi$ and $\Phi$ are, respectively, totally antisymmetric and symmetric wavefunctions. This transformation can be identified to an element of the unitarty group $U(N)$ and should be written in terms of a suitable function $\Theta$. This is

$$
U=e^{-i \Theta\left(z_{1}, \cdots, z_{N}\right)} .
$$

Clearly from (58) we have some information about $\Theta$. Indeed, it should be defined such that an interchange of a pair of variables gives the constraint

$$
\exp \left[-i \Theta\left(z_{1}, \cdots, z_{i}, \cdots, z_{j}, \cdots, z_{N}\right)\right]=-\exp \left[-i \Theta\left(z_{1}, \cdots, z_{j}, \cdots, z_{i}, \cdots, z_{N}\right)\right] .
$$

Consequentely, $\Theta$ should be realized in such way that (60) must be fullfiled. Thus, we map $\Theta$ in terms of our language as

$$
\Theta\left(z_{1}, \cdots, z_{N}\right)=\eta \sum_{i<j} \operatorname{Im} \ln \left(z_{i}-z_{j}\right)
$$

This function is not only interesting in the present case but also in the field theory. In fact, it can be linked to the relative angle between the position vectors the $i^{t h}$ and $j^{\text {th }}$ particles. This is

$$
\Theta\left(z_{1}, \cdots, z_{N}\right)=(\eta-1) \sum_{j \neq i}^{M} \theta_{i j}
$$


where $\theta_{i j}$ is related to the Chern-Simons potential of $i^{t h}$ particle through the relation

$$
A_{i}^{\mathrm{CS}}=(\eta-1) \vec{\nabla}_{i} \sum_{j \neq i}^{M} \theta_{i j}=(\eta-1) \sum_{j \neq i}^{M} \frac{\vec{e}_{z} \times\left(\vec{r}_{i}-\vec{r}_{j}\right)}{\left|\vec{r}_{i}-\vec{r}_{j}\right|^{2}}
$$

which was used to study the anyon systems. This mapping can offer another way to talk about the interacting particles in terms of the Dirac formalism. Also one could think to a make matrix model analysis of the present system by adopting the Susskind-Polychronakos approach [22, 23].

Now we have all ingredients to do our task. Indeed, since (58) is a singular gauge transformation, the Hamiltonian for $\Phi$ can be obtained from (57) for $\Psi$. Indeed, we show that

$$
H_{\mathrm{D}}^{\mathrm{int}}=U^{\dagger} H_{\mathrm{D}} U
$$

With this, we are now able to generate a specific potential that governs the interaction between particles. More precisely, is not hard to end up with the Dirac Hamiltonian for interacting particles in the presence of a magnetic field, which is

$$
H_{\mathrm{D}}^{\mathrm{int}}=\frac{i}{\sqrt{2}}\left(\begin{array}{cc}
0 & \sum_{i}^{M}\left(-2 \frac{\partial}{\partial_{\bar{z}_{i}}}+\frac{B}{2} z_{i}\right)-2 \eta \sum_{i, j \neq i}^{M} \frac{1}{\bar{z}_{i}-\bar{z}_{j}} \\
-\sum_{i}^{M}\left(2 \frac{\partial}{\partial_{z_{i}}}+\frac{B}{2} \bar{z}_{i}\right)+2 \eta \sum_{i, j \neq i}^{M} \frac{1}{z_{i}-z_{j}} & 0
\end{array}\right) .
$$

This form shows how the gauge transformation can be employed to produce some kind of interaction from the free case. Moreover, the above mapping tells us that the spectrum and corresponding eigenfunctions of $H_{\mathrm{D}}^{\text {int }}$ can be deduced from those of $H_{\mathrm{D}}$. For instance, we have a similar relation to (58) between the states $\left|m_{i}, n_{i}\right\rangle$ for $M$-particles those are tensor products of single states (14) 17) and $\left|\left\{m_{i}\right\}, \eta\right\rangle_{\mathrm{D}}$.

We conclude this part by noting that the involved gauge transformation can be generalized to higher order in terms of some interactions. This can be done by considering more that three-body correlations and therefore offers different integrability of some models. This will be interesting in the FQHE world because recall that this is based on such interactions to be understood.

\section{Composite fermion wavefunction}

To give an explanation of the filling factors beyond the Laughlin states, Jain has introduced the composite fermion formalism [14]. In fact, they are new kind of particles appeared in condensed matter physics to provide an explanation of the behavior of particles moving in the plane when a strong magnetic field $B$ is present. Particles possessing $2 p$, with $p \in N^{*}$, flux quanta (vortices) can be thought of being composite fermions. One of the most important features of them is they feel effectively a magnetic field of the form

$$
B^{*}=B \pm 2 p \Phi_{0} \rho
$$

where in our convention the unit flux is $\Phi_{0}=2 \pi$. Recalling the relation between the filling factor $\nu$ and $B$, one can define a similar quantity for the field $B^{*}$. This can be written as

$$
\nu^{*}=2 \pi \frac{\rho}{B^{*}} .
$$


It is clear that from (66), the factors $\nu$ and $\nu^{*}$ can be linked to each other through

$$
\nu=\frac{\nu^{*}}{2 p \nu^{*} \pm 1} \text {. }
$$

This relation has been used to deal with different issues related to QHE in 2DEG. More discussions about the mapping (68) and its applications can be found in [14].

As an immediate consequence of (68), we can map the anomalous FQHE in terms of IQHE in graphene. Indeed, it is easy to see

$$
\nu^{\mathrm{G}}=2 \frac{2 n+1}{4 p(2 n+1) \pm 1} .
$$

This result has been obtained in different contexts, for instance see [19]. It is obvious that for $n=0$, we obtain Laughlin states and the anomalous IQHE can be recovered by fixing $p=0$. Moreover, (69) tells us that the Jain's series is quite different from the 2DEG. Therefore, it is interesting to focus on FQHE in graphene.

Remembering the Laughlin potential established before, it is natural to ask about that behind $\nu^{\mathrm{G}}$. To reply this question, we build a wavefunction corresponding to $\nu^{\mathrm{G}}$ in terms of a generalized potential. In the beginning, let us see how CF picture does look like for the Laughlin states, which are the same for both systems: semiconductors and graphene. Indeed, following Jain idea [14], one can write the Laughlin wavefunction as

$$
\psi_{\text {Laugh }}^{p}(z, \bar{z})=\prod_{i<j}^{M}\left(z_{i}-z_{j}\right)^{2 p} \chi_{1} \exp \left(-\frac{B}{4} \sum_{i}^{M}\left|z_{i}\right|^{2}\right)
$$

where $\chi_{1}$ is the wavefunction of the fully occupied state $\nu=1$. Now it is clear that for higher Landau level, one may generalize (70) to that of the form

$$
\psi_{\text {Jain }}^{p, n}(z, \bar{z})=\prod_{i<j}^{M}\left(z_{i}-z_{j}\right)^{2 p} \chi_{2(2 n+1)} \exp \left(-\frac{B}{4} \sum_{i}^{M}\left|z_{i}\right|^{2}\right)
$$

and $\chi_{2(2 n+1)}$ is describing the composite fermions at the anomalous integer filling factor $\nu_{\mathrm{cf}}$. This is a obvious mapping between the particles generating FQHE in graphene and composite fermions behind unconventional integer QHE.

At this level, one may ask about the potential of interaction that is responsible for the emergence of $\nu^{G}$ and therefore the model that describes such case. One way to answer this inquiry is to make a straightforward generalization of the Laughlin potential (29-30). Then by analogy, we can define the Jain potential as

$$
\left(V_{z}^{\text {Jain }}\right)_{i}=-2 i \frac{\partial}{\partial z_{i}}\left(\ln F^{\text {Jain }}-\ln \bar{F}^{\text {Jain }}\right), \quad\left(V_{\bar{z}}^{\text {Jain }}\right)_{i}=2 i \frac{\partial}{\partial \bar{z}_{i}}\left(\ln F^{\text {Jain }}-\ln \bar{F}^{\text {Jain }}\right) .
$$

Clearly, for $n=0$ we recover (29) function $F^{\text {Jain }}(z, \bar{z})$ as

$$
\psi_{\text {Jain }}^{p, n}(z, \bar{z})=F^{\text {Jain }}(z, \bar{z}) \exp \left(-\frac{B}{4} \sum_{i}^{M}\left|z_{i}\right|^{2}\right) .
$$

Of course it should be described by some Hamiltonian that involves $\left(V_{z}^{\text {Jain }}\right)_{i}$ and $\left(V_{\bar{z}}^{\text {Jain }}\right)_{i}$ as important ingredients. This requires a deep attention because of the complexity of $\psi_{\text {Jain }}^{p, n}(z, \bar{z})$ and therefore needs to be done separately. Also another important point that is one could think about a relation between these potentials and those of Chern-Simons in similar way to the Laughlin case. 


\section{$6 \quad S U(N)$ wavefunctions}

Using different theoretical arguments, some authors suggested a possible FQHE in graphene. As we have seen before, this will be natural if we look at the anomalous IQHE as a product of collective behavior of the composite fermions instead of particles. Moreover, different wavefunctions have been proposed to describe the anomalous FQHE for different filling factors. Among them, we cite that constructed by Goerbig and Regnault [12] to solve some problems brought by other theories like for instance that developed in [9]. However, this wavefunction is sharing many common features with our early proposal [24]. On the other hand, the Goerbig and Regnault states are a direct extended version of those of the Laughlin [18] as well as Halperin [15]. To get more advantages, let us return back to the constructed $S U(N)$ wavefunctions [13] describing the anomalous FQHE at the filling factor

$$
\nu=q_{i} K_{i j}^{-1} q_{j}
$$

where $K_{i j}$ is an $N \times N$ matrix and $q_{i}$ is a vector. In fact, this included different fractions suggested recently for FQHE in graphene and allowed to make contact with different proposals.

We start by reviewing the building of such wavefunctions. Indeed, to construct a general wavefunction, we use the obtained result so far. In doing so, we consider two sectors labeled by $(m)$ and $(n)$. This is the case for instance in graphene where there are two subsystems forming a honeycomb. Le us define $\psi^{(m, n)}$ as a tensor product

$$
\psi^{(m, n)}=\psi^{(m)} \otimes \psi^{(n)}
$$

where each $\psi^{(m)}$ is given in (17). Assuming that the condition between matrix elements $K_{i j}=K_{j i}$ is fulfilled, a natural way to construct the required wavefunction is

$$
\begin{aligned}
|\Psi\rangle_{\text {Jel }}= & \prod_{m=1}^{N}\left[\epsilon^{i_{1} \cdots i_{M_{m}}} \psi_{i_{1}}^{(m)} \psi_{i_{2}}^{(m)} \cdots \psi_{i_{M_{m}}}^{(m)}\right]^{K_{m m}-K_{m n}} \prod_{n=1}^{N}\left[\epsilon^{j_{1} \cdots j_{M_{n}}} \psi_{i_{1}}^{(n)} \psi_{i_{2}}^{(n)} \cdots \psi_{i_{M_{n}}}^{(n)}\right]^{K_{n n}-K_{m n}} \\
& \prod_{m<n}^{N}\left[\epsilon^{k_{1} \cdots k_{M_{m}+M_{n}}} \psi_{k_{1}}^{(m, n)} \psi_{k_{2}}^{(m, n)} \cdots \psi_{i_{M_{m}+M_{n}}}^{(m, n)}\right]^{K_{m n}}|0\rangle .
\end{aligned}
$$

It is not hard to get the constraint

$$
L_{\text {tot }}|\Psi\rangle_{\text {Jel }}=0
$$

where the total angular momenta is given by

$$
L_{\mathrm{tot}}=\sum_{i}^{N} L_{i}
$$

This wavefunction is actually capturing some interesting effect that is relevant to discuss about FQHE in graphene. Indeed, the following term

$$
\prod_{m<n}^{M}\left[\epsilon^{k_{1} \cdots k_{M_{m}+M_{n}}} \psi_{k_{1}}^{(m, n)} \psi_{k_{2}}^{(m, n)} \cdots \psi_{i_{M_{m}+M_{n}}}^{(m, n)}\right]^{K_{m n}}
$$

is nothing but is an inter-layer correlation. In conclusion, our configuration could be a good ansatz for the ground states of FQHE in graphene. This will be clarified soon. 
At this level, we have all ingredients to do our job. Indeed, we start by defining a new complex variable

$$
\zeta_{i}= \begin{cases}z_{i}^{(m)} & \text { for } i=1, \cdots, M \\ z_{i-M}^{(n)} & \text { for } i=M+1, \cdots, 2 M\end{cases}
$$

assuming that the particle numbers are equal, i.e. $M_{1}=M_{2}=M$, and recalling the antisymmetric Vandermonde determinant for the fully occupied state

$$
\prod_{i<j}\left(z_{i}-z_{j}\right)=\operatorname{det}\left(z_{i}^{M-j}\right)=\epsilon^{i_{1} \cdots i_{M}} z_{i_{1}}^{0} \cdots z_{i_{M}}^{M-1}
$$

Therefore, (76) can be projected on the complex plane as

$$
\begin{aligned}
\Psi_{\mathrm{Jel}}= & \prod_{m=1}^{N}\left[\epsilon^{i_{1} \cdots i_{M}}\left(z_{i_{1}}^{(m)}\right)^{0} \cdots\left(z_{i_{M}}^{(m)}\right)^{M-1}\right]^{K_{m n}-K_{m n}} \prod_{n=1}^{N}\left[\epsilon^{j_{1} \cdots j_{M}}\left(z_{j_{1}}^{(n)}\right)^{0} \cdots\left(z_{j_{M}}^{(n)}\right)^{M-1}\right]^{K_{n n}-K_{m n}} \\
& \prod_{m<n}^{N}\left[\epsilon^{k_{1} \cdots k_{2 M}} \zeta_{k_{1}}^{0} \cdots \zeta_{k_{2 M}}^{2 M-1}\right]^{K_{m n}} \Psi_{0}
\end{aligned}
$$

It can be written in the standard form as

$$
\Psi_{\mathrm{Jel}}=\prod_{m=1}^{N} \prod_{i<j}^{M}\left(z_{i}^{(m)}-z_{j}^{(m)}\right)^{K_{m m}} \prod_{n=1}^{N} \prod_{i<j}^{M}\left(z_{i}^{(n)}-z_{j}^{(n)}\right)^{K_{n n}} \prod_{m<n}^{N} \prod_{i, j}^{M}\left(z_{i}^{(m)}-z_{j}^{(n)}\right)^{K_{m n}} \Psi_{0} .
$$

This exactly coincides with that constructed by Goerbig and Regnault [12] and what we have proposed [24] in terms of the matrix model and non-commutative Chern-Simons theories. The wavefunction (83) is a good candidate for describing the anomalous FQHE in graphene. As it is seen in [13, the present wavefunction is general and has many features. In particular, it can be fixed to recover different filling factors like for instance those in [15, 25] as well as others. Note that, the above wavefunctions are the planar limit of those constructed on two-sphere [13].

As far as the graphene is concerned, we restrict our analysis to the case $N=4$. In fact, we give an explicit realization of the Jain potential and therefore the Hamiltonian that captures such interactions. In doing so, we can use the same procedure as for the Laughlin case to write down the potential behind (83). For instance, the first term in (83) is originated from

$$
\left[\left(V_{z}^{\mathrm{Jel}}\right)_{i}\right]_{K_{K_{m m}}}=-2 i \frac{\partial}{\partial z_{i}}\left(\ln F_{K_{m m}}^{\mathrm{Jel}}-\ln \bar{F}_{K_{m m}}^{\mathrm{Jel}}\right), \quad\left[\left(V_{\bar{z}}^{\mathrm{Jel}}\right)_{i}\right]_{\left.\right|_{K_{m m}}}=2 i \frac{\partial}{\partial \bar{z}_{i}}\left(\ln F_{K_{m m}}^{\mathrm{Jel}}-\ln \bar{F}_{K_{m n}}^{\mathrm{Jel}}\right)
$$

and the potential corresponding to the second term can be obtained in similar way, i.e. changing $K_{m m}$ by $K_{n n}$. However, the inter-layer interaction is

$$
\left[\left(V_{z}^{\mathrm{Jel}}\right)_{i}\right]_{\left.\right|_{K m n}}=-2 i \frac{\partial}{\partial z_{i}}\left(\ln F_{K_{m n}}^{\mathrm{Jel}}-\ln \bar{F}_{K_{m n}}^{\mathrm{Jel}}\right), \quad\left[\left(V_{\bar{z}}^{\mathrm{Jel}}\right)_{i}\right]_{K_{K_{m n}}}=2 i \frac{\partial}{\partial \bar{z}_{i}}\left(\ln F_{K_{m n}}^{\mathrm{Jel}}-\ln \bar{F}_{K_{m n}}^{\mathrm{Jel}}\right) .
$$

These can be worked as for the previous cases to get the corresponding wavefunctions. In doing so, we end up with the form

$$
\psi_{\mathrm{Jel}}^{K_{m m}, K_{m n}}(z, \bar{z})=\prod_{m=1}^{4} F_{K_{m m}}^{\mathrm{Jel}} F_{K_{n n}}^{\mathrm{Jel}} F_{K_{m n}}^{\mathrm{Jel}} \Psi_{0}
$$


Of course this is associated to a Hamiltonian, which involves such potentials. Indeed, this can be obtained as two copies of $H_{\mathrm{L}}^{\mathrm{int}}$ amended by inter-layer correlation. It can straightforwardly be generalized to include other kind of interaction, in particular more that three-body type.

The above wavefunctions has many features. Indeed, it can be linked to the composite Dirac fermions and derive some interesting results. To clarify this point, we return to the definition of the filling factor and write for any $j$ the relation

$$
\nu_{j}=\frac{\rho_{j}}{N_{B}}
$$

where the number $N_{B}$ is the quantized flux. In our case, it can be written in terms of elements of the matrix $K(\underline{74})$ as

$$
N_{B}=\rho_{j} K_{j}+\sum_{i \neq j} \rho_{i} K_{i j}
$$

and we have set $K_{j j}=K_{j}$. This offers different discussions about the above wavefunctions and its links to other proposals. For instance, we can establish a bridge between the filling factor (74) and that coresponds to the composite Dirac fermions. For this, let us start by identifying (69) and (74) to see what are the consequences. Clearly, for $n=0$ we end up with the Laughlin series, namely

$$
\frac{1}{K_{i j}}=\frac{2}{4 p \pm 1}
$$

which is in agreement with different investigations [11, 13]. However, the interesting case is when $p=0$ that leads to the relation

$$
2 \sum_{i \neq j} \frac{\rho_{i}}{\rho_{j}} K_{i j}= \pm\left(\frac{1}{2 n+1}-1\right) .
$$

This offers different discussions about the ratio $\frac{\rho_{i}}{\rho_{j}}$ and in particular its quantization. For this, let us explicitly evaluate the involved series, which can be done by recalling a similar formula. This is [26]

$$
\sum_{l=1}^{m} \frac{(-1)^{k+1}}{k+1} \frac{m !}{k !(m-k) !}=\frac{1}{m+1}-1
$$

Therefore, by comparing both series we can identify $2 n$ to $m$ and more importantly the ration can be quantized as follows

$$
\frac{\rho_{i}}{\rho_{j}} \sim \frac{(-1)^{k+1}}{2(k+1)} \frac{2 n !}{k !(2 n-k) !} K_{i j}^{-1} .
$$

This mapping shows how the present theory can be restricted to make a possible connection with the composite Dirac fermions. Moreover, other theories can be recovered from our the present results like for instance that proposed in the reference [11]. On the other hand, some discussions can be added about the nature of the Hall droplet in terms of the ratio and in particular the quantization of its area.

\section{Conclusion}

The present work was devoted to analyze the behaviour of an interacting system in the presence of an external magnetic field. More precisely, we have focused on a specific case where the system is 
exactly a sheet of graphene. To do this, we have started by considering a model that captures a kind of interaction, which applied before at different occasions to discuss the conventional QHE. Before doing so, we have presented some discussions about the effective potential that corresponds to the Laughlin wavefunction at the filling factor $\nu=\frac{1}{2 l+1}$. This was a typical example of a generic case that is the Jain wavefunction.

By considering a Hamiltonian of the present system where the interaction is restricted to be two and three-body types, we have employed a previous technical to achieve our goal. This was done by making use the mapping between three Hamiltonian's: Landau, Pauli-Schrödinger and Dirac. This means that we have derived an appropriate Dirac operator in terms of the involved interaction. The mapping allowed us to get in a simple way different spectrum's entering in the game and the excited states as well. Using a gauge transformation, we have established a link between the free and interacting Dirac operators. This can be generalized to take into account of other interaction types.

As concerning QHE in graphene, in the beginning we have extended the Laughlin potential to the Jain case. Indeed, we have generalized the interaction terms that are behind the series $\nu=\frac{1}{2 l+1}$ to that for the composite fermions in graphene, i.e. $\nu^{G}$. This was shown another way to talk about the Jain wavefunction for graphene and its corresponding effective potential. Of course these can be used to deal with other proposals related to different wavefunctions suggested for the anomalous FQHE.

As second part, we have concentrated on the $S U(N)$ wavefunction that captures different filling factors and generalizes some others. In the first step, we have given the corresponding potentials as an explicit example to illustrate our general procedure for Jain wavefunction. To explicitly show its relation to some theories, we have established its link to the composite fermion pictures. This has been done by identifying both of filling factors. As consequence, we have found a quantized term that include the density of interacting particles. This in principle gave rise to another way to interpret our results and made contact with others.

The obtained results suggest to investigate and deal with other issues related to QHE in graphene. As example, we are now focussing on a second problem that involves another type of interaction. More precisely, we are wondering to understand the behaviour of the confined relativistic particles in the presence of an external magnetic field.

\section{Acknowledgment}

This work was completed during a visit of AJ to the Abdus Salam Centre for Theoretical Physics (Trieste, Italy) in the framework of junior associate scheme. He would like to acknowledge the financial support of the centre.

\section{References}

[1] For instance see R.E. Prange and S.M. Girvin (editors), "The Quantum Hall Effect", (Springer, New York 1990).

[2] K.S. Novoselov, A.K. Greim, S.V. Morosov, D. Jiang, M.I. Katsnelson, V.I. Grigorieva, L. Levy, S.V. Dubonos and A.A. Firsov, Nature 438 (2005) 197. 
[3] Y. Zhang, Y.-W. Tan, H.L. Störmer and P. Kim, Nature 438 (2005) 201.

[4] Y. Zheng and T. ando, Phys. Rev. B65 (2002) 245420.

[5] V.P. Gusynin and S.G. Sharapov, Phys. Rev. Lett. 95 (2005) 146801.

[6] D.C. Tsui, H.L. Stormer, and A.C. Gossard, Phys. Rev. Lett. 48 (1982) 1559.

[7] K. Yang, S. Das Sarma and A.H. MacDonald, Phys. Rev. B74 (2006) 075423.

[8] M.O. Goerbig and N. Regnault, Phys. Rev. B74 (2006 ) 161407.

[9] C. Töke, P.E. Lammert, J.K. Jain and V.H. Crespi, Phys. Rev. 74 (2006) 235417.

[10] D.V. Khveshchenko, Phys. Rev. B75 (2007) 153405.

[11] C. Töke and J.K. Jain, Phys. Rev. B75 (2007) 244540, cond-mat/0701026.

[12] M.O. Goerbig and N. Regnault, Phys. Rev. B75 (2007) 241405, cond-mat/0701661.

[13] A. Jellal, Nucl. Phys. B804 (2008) 361, arXiv:0709.4126.

[14] J.V. Jain, Phys. Rev. Lett. 63 (1989) 199; Phys. Rev. B41 (1990) 7653; Adv. Phys. 41 (1992) 105; O. Heinonen (editor), Composite Fermions: A Unified View of Quantum Hall Regime, (World Scientific, 1998).

[15] B.I. Halperin, Helv. Phys. Acta. 56 (1983) 75.

[16] D. Karabali and B. Sakita, Int. J. Mod. Phys. A6 (1991) 5079; D. Karabali, Collective Field Representation of Nonrelativistic Fermions in (1+1) Dimensions, hep-th/9109013.

[17] R.K. Ghosh and S. Rao, Int. J. Mod. Phys. B12 (1998) 1125, cond-mat/9703217.

[18] R.B. Laughlin, Phys. Rev. Lett. 50 (1983) 1395.

[19] N.M.R. Peres, F. Guinea and A.H. Castro Neto, Phys. Rev. B73 (2006) 125411.

[20] A. Jellal, Nucl. Phys. B (2005) 554, hep-th/0505095.

[21] M. Koshino and T. Ando, Phys. Rev. B75 (2007) 235333, cond-mat/0705.2322.

[22] L. Susskind, The Quantum Hall Fluid and Non-Commutative Chern Simons Theory, (2001) hep-th/0101029.

[23] A.P. Polychronakos, JHEP 0104 (2001) 011, hep-th/0103013.

[24] A. Jellal and M. Schreiber, J. Phys. 37: Math. Gen. (2004) 3147.

[25] D. Yoshioka, A.H. MacDonald and S.M. Girvin, Phys. Rev. B39 (1989) 1932.

[26] I.S. Gradshteyn and I.M. Ryzhik, "Table of Integrals, Series and Products", (Academic Press, Orlando 1980). 